\title{
Mastitis en vacas Holstein y factores asociados en tambos de Córdoba, Argentina
}

\author{
Vissio, C. ; Pereyra, M.'; Lamberti, M. ${ }^{2}$; Bonetto, C. ${ }^{2}$; Larriestra, A. ${ }^{1,2}$ \\ ${ }^{1}$ CONICET, Univ. Nac. Río Cuarto, Córdoba, Argentina. \\ ${ }^{2}$ IAPCBYA, Univ. Nac. Villa María, Córdoba, Argentina. \\ E-mail: cvissio@ayv.unrc.edu.ar
}

\begin{abstract}
Resumen
Vissio, C.; Pereyra, M.; Lamberti, M.; Bonetto, C.; Larriestra, A.: Mastitis en vacas Holstein y factores asociados en tambos de Córdoba, Argentina. Rev. Vet. 31: 2, 120-125, 2020. El objetivo de este trabajo fue estimar la incidencia de mastitis clínica (MC) en tambos e identificar factores individuales asociados a nuevos episodios. Nueve tambos del tipo semipastoril que aplicaban medidas de control y prevención de la mastitis, fueron incluidos y visitados dos veces con un intervalo de 15 días. Una muestra aleatoria de vacas fue seleccionada en la primera visita y monitoreada durante los 15 días posteriores para la identificación de los nuevos casos de MC. La incidencia fue determinada como el cociente entre el número de casos nuevos de $\mathrm{MC}$ durante el período de seguimiento y el número de vacas sanas en la primera visita. Los factores de riesgo evaluados a nivel de vaca fueron: suciedad de la ubre, distancia al piso de la punta del pezón anterior, número ordinal de partos y días en lactancia. A nivel de cuarto se evaluó la presencia de pérdida espontánea de leche, condición del pezón considerando estado de la piel, presencia de anillo e hiperqueratosis. El grado de mastitis subclínica se valoró utilizando el California Mastitis Test (CMT). Un modelo lineal generalizado mixto fue ajustado para evaluar la asociación entre los factores relevados y la presentación de un caso nuevo de MC. Un total de 990 vacas fueron analizadas identificándose 62 (6,7\%, intervalo de confianza-IC $95 \% 5,0-8,3)$ casos nuevos de MC. Los factores asociados a la presentación de un caso nuevo de mastitis clínica en el modelo final fueron días en lactancia, suciedad de la ubre y grado de CMT. Vacas con ubres muy sucias (grado 4) y resultado de $\mathrm{CMT} \geq 2$ en al menos un cuarto, mostraron valores de odds ratio (OR) de $2,56(1,19-5,50)$ y $3,29(1,5-7,2)$, respectivamente. Los atributos de la vaca identificados como asociados pueden ser indicadores de riesgo para el manejo especifico de la salud mamaria en el tambo.
\end{abstract}

Palabras clave: vacas, California mastitis test, higiene de ubre, condición del pezón.

\begin{abstract}
Vissio, C.; Pereyra, M.; Lamberti, M.; Bonetto, C.; Larriestra, A.: Mastitis in Holstein cows and associated factors in dairy farms in Córdoba, Argentina. Rev. Vet. 31: 2, 120-125, 2020. The objective of this study was to estimate the incidence of clinical mastitis (CM) in dairy farms and identify individual factors associated with these new episodes. Nine semipastoral dairy farms applying mastitis control and prevention measures were included and visited twice with an interval of 15 days. A random sample of cows was selected in the first visit and monitored during the following 15 days for the identification of new cases of CM. The incidence was determined as the ratio between the number of new CM cases during the follow-up period and the number of healthy cows at the first visit. The risk factors evaluated at cow level were udder hygiene, distance to the floor of the anterior quarter teat, parity and days in milk; at quarter level was evaluated the leaking milk, teat skin condition, presence of ring and hyperkeratosis and subclinical mastitis using the California Mastitis Test (CMT). A generalized mixed linear model was adjusted to evaluate the association between the factors surveyed and a new CM case. A total of 990 cows were analyzed and $62(6.7 \%, 95 \%$ CI 5.0 8.3) new cases of $\mathrm{CM}$ were identified. The factors associated to a new case of clinical mastitis in the final model were days in milking, udder hygiene and CMT level. Cows with very dirty udders (level 4) and CMT result $\geq 2$ in at least one quarter showed odds ratio (OR) values of
\end{abstract}


2.56 (1.19-5.5) and $3.29(1.5-7.2)$, respectively. Cow attributes identified as associated may be risk indicators for specific mammary health management in the dairy herd.

Key words: cows, California mastitis test, udder hygiene, teat condition.

\section{INTRODUCCIÓN}

La producción de leche en Argentina en el año 2018, según datos del Ministerio de Agroindustria de la Nación, alcanzó los 10.527 millones de litros, siendo las provincias de Córdoba, Santa Fe y Buenos Aires las principales productoras de leche.

La mastitis bovina es una de las enfermedades con mayor impacto económico en los rodeos lecheros ${ }^{9}$, por lo cual la reducción de los niveles de dicha afección representa un desafío en los tambos de Argentina para mejorar la productividad y moderar los costos asociados.

La incidencia de mastitis clínica está asociada con múltiples factores de riesgo a nivel de rodeo y a nivel individual. La implementación de programas de salud mamaria en los tambos permite reducir la exposición a factores de riesgo de rodeo y mejorar los índices de salud en general.

La identificación de factores de riesgo a nivel individual permitiría detectar tempranamente a vacas con mayor predisposición a desarrollar mastitis, y podría ser la base para implementar estrategias de prevención secundaria que disminuyan la diseminación de la enfermedad y las demás consecuencias negativas de un caso clínico.

Entre los factores individuales se identifican los de vaca y los de cuarto. Entre los primeros el número ordinal de parto, el estadio de lactancia y la historia de mastitis clínica y subclínica son los más reconocidos ${ }^{4,15,16,24}$. La posición del pezón, el grado de hiperqueratosis y la distancia al piso son factores identificados a nivel de cuarto ${ }^{7,10,13}$. Considerando los escasos reportes en Argentina respecto de la identificación de factores individuales asociados a mastitis clínica, el conocimiento de las mismos podría ayudar al desarrollo de prácticas de manejo de la salud mamaria acorde a nuestras condiciones de producción.

El objetivo de este trabajo fue estimar la incidencia de mastitis clínica en tambos del tipo semi-pastoriles de la Provincia de Córdoba (Argentina) e identificar factores asociados a estos nuevos episodios.

\section{MATERIAL Y MÉTODOS}

Población de estudio. Esta investigación involucró la evaluación de 990 vacas en ordeñe pertenecientes a 9 tambos de la Provincia de Córdoba. El criterio de inclusión de los establecimientos consistió en que fueran del tipo semi-pastoril y que aplicaran habitualmente el plan de los 5 puntos de control de la mastitis ${ }^{12}$. Los tambos fueron visitados dos veces con un intervalo de 15 días entre los meses de marzo y agosto del año 2016 y a su vez se realizó el seguimiento de las vacas seleccionadas durante el lapso de tiempo entre las dos visitas.

Riesgo de mastitis clínica. En la primera visita a cada tambo, se identificaron los casos de mastitis clínica (MC) en una muestra aleatoria de vacas. La cantidad de vacunos a seleccionar en cada establecimiento fue estimada asumiendo una prevalencia de mastitis del 5\%, una precisión absoluta del 3\% y una confianza del $95 \%{ }^{25}$ implementadas en el programa EPIDAT 3.1 (Xunta de Galicia, OPS. 2006).

Las vacas a examinar fueron seleccionadas mediante un procedimiento al azar sistemático, para esto, el total de vacas en ordeño al momento de la visita fue dividido por el tamaño de la muestra establecido previamente. De esta manera se identificó el intervalo de selección, para posteriormente elegir al azar la primera vaca ${ }^{25}$

La identificación de los casos de MC fue realizada a nivel de cuarto al momento del realizar el despunte (descarte de los primeros chorros de leche) de cada vaca antes del ordeñe. Un cuarto mamario fue considerado con mastitis clínica si se observaba alteración de la leche (grumos, sangre, aspecto aguado) asociado o no a signos locales (dolor, enrojecimiento, calor en el cuarto) o sistémicos (decaimiento, fiebre) ${ }^{14}$.

En la segunda visita al tambo, las vacas seleccionadas previamente fueron revisadas para determinar la presencia de un caso nuevo de mastitis clínica. Además, a partir de las planillas de registro acordadas con el personal a cargo del ordeñe, se identificaron las vacas que durante el período de 15 días habían presentado un episodio de MC.

De esta manera, un caso nuevo de mastitis clínica fue definido como aquella vaca que estando sana en la primera visita, desarrolló una mastitis clínica en los 15 días subsecuentes ${ }^{20}$. Cualquier episodio de MC ocurrido dentro de las dos semanas posteriores al evento de MC fue considerado como el mismo caso ${ }^{8}$.

Evaluación de factores de riesgo. Potenciales predictores a nivel de vaca y de cuarto fueron relevados en cada uno de los animales seleccionados para este estudio durante la primera visita. Los factores evaluados a nivel de vaca fueron: suciedad de la ubre en una escala de 1 a $4{ }^{23}$, distancia al piso de la punta del pezón anterior derecho o izquierdo respecto de la altura del corvejón de la vaca (por encima, a la misma altura o por debajo del corvejón), número ordinal de parto (vacas de un parto y vacas de 2 o más partos) y días en lactancia.

Los potenciales factores de riesgo evaluados a nivel de cuarto fueron: presencia de pérdida espontánea de leche ${ }^{18}$, condición del pezón considerando estado de 
la piel (seca, muy seca y agrietada), presencia de anillo (leve, moderado o extremo) e hiperqueratosis (leve, moderada o extrema en la punta del pezón) ${ }^{6,13}$. Además, en la primera visita se realizó la determinación de California Mastitis Test (CMT) considerando tres niveles de positividad: traza, grado $1 \mathrm{y}$ grado 2 .

Análisis estadístico. Un análisis descriptivo fue realizado a nivel de cuarto y de vaca. A nivel de vaca, la presencia de mastitis clínica fue definida como la presencia de al menos un cuarto afectado. De forma análoga, los atributos de cuarto fueron agregados a nivel de vaca realizando también una interpretación en paralelo, considerando expuesta a la vaca que presentara el atributo en al menos uno de sus cuartos. Un análisis univariado a nivel de vaca fue realizado para evaluar la asociación entre cada factor relevado y la presencia de al menos un cuarto con mastitis clínica, utilizando el test de Chi cuadrado ${ }^{25}$.

Posterior al análisis univariado, se procedió a ajustar un modelo lineal generalizado mixto en InfoStat ${ }^{5}$. La variable respuesta fue un caso nuevo de MC identificado durante el período de seguimiento y las variables independientes fueron los factores que presentaban un valor de $\mathrm{p} \leq 0,25$ en el análisis univariado.

El número de partos y los días en lactancia fueron forzados en el modelo considerando su potencial efecto confundidor. Las variables relacionadas a la condición de la piel y punta de pezón fueron clasificadas en dos categorías $(0$ : ningún pezón de la vaca mostraba alteración o lesión y 1: al menos un pezón de la vaca mostraba lesión). El tambo fue incluido en el modelo como efecto aleatorio.

Las interacciones plausibles biológicamente fueron incluidas en el modelo para su evaluación. El nivel de significancia considerado en el modelo final fue un valor de $\mathrm{p}<0,05$. Los resultados del modelo fueron presentados como odds ratio (OR) con sus respectivos intervalos de confianza del 95\% (IC 95\%). La fracción atribuible poblacional (FAP) fue estimada para los predictores asociados en el modelo final ${ }^{11}$.

\section{RESULTADOS}

Un total de 990 vacas (3886 cuartos) fueron analizadas (Tabla 1). Cada tambo $(\mathrm{n}=9)$ aportó -en promedio- un $11,1 \%$ de las observaciones (rango $=6,7-14,5$ ). En la primera visita fueron identificadas $45(4,5 \%$, IC $95 \% 3,2-5,9)$ vacas con al menos un cuarto con MC. El total de casos nuevos de MC identificados durante el período de seguimiento de las vacas seleccionadas fue $62(6,7 \%$, IC $95 \% 5,0-8,3)$.

El 33,0\% de las vacas estudiadas eran vaquillonas de primer parto, el resto eran vacas con 20 más partos y el promedio de días en lactancia fue 192 (desvío stan- dard DS $=133$ ). Entre los tambos estudiados el rango promedio de días en lactancia fue 132-237.

Entre los otros factores evaluados a nivel de vaca, se observó un $41,2 \%$ de ubres limpias (grado de suciedad 1) con un rango entre los tambos de $2,8 \%-67,4 \%$ y un $22,1 \%$ de vacas con la punta del pezón anterior por debajo del corvejón con un rango entre los tambos de $0,0 \%-35,4 \%$. Los factores agregados a nivel de vaca y su asociación con la presentación de un nuevo caso de mastitis clínica, se muestran en la Tabla 2.

Los factores asociados a la presencia de un caso nuevo de mastitis clínica retenidos en el modelo final fueron: días de lactancia, suciedad de la ubre y grado de CMT, como indica la Tabla 3. La fracción atribuible poblacional estimada para suciedad de la ubre y grado de CMT fue $17,7 \%$ y $39 \%$, respectivamente.

\section{DISCUSIÓN}

La frecuencia de MC ha sido investigada previamente en rodeos lecheros de Argentina (ArCh Med Vet 47: 7-14), con prevalencias estimadas de $3 \%$ (IC $95 \%$ $2,3-3,7$ ) y $2,5 \%$ (IC $95 \% 1,9-3,1)$, valores levemente inferiores a los hallados en este estudio durante la primera visita al predio $(4,5 \%$; IC $95 \% 3,2-5,9)$. En relación a la incidencia de MC, lo reportado en este estudio $(6,7 \%$; IC $95 \% 5,0-8,3)$ fue superior a lo publicado previamente $(3,9 \%$; IC $95 \% 3,1-4,7)$, siendo esta comparación válida debido a que la metodología en la estimación de los casos nuevos de MC fue similar ${ }^{20}$.

Tal incremento en el número de casos de mastitis puede deberse a múltiples factores involucrando tanto las características de las vacas como el ambiente y las prácticas de manejo. Los sistemas de manejo incluidos en el trabajo previo realizado en Argentina, incluyó todo tipo de sistemas, desde pastoriles hasta totalmente confinados, y esto podría explicar en parte las diferencias encontradas ${ }^{20}$

El grado de suciedad de ubre es un indicador de la limpieza de las vacas y del ambiente en el tambo 1,2,3,19, y esta variable ha sido asociada con el recuento de células somáticas y la presencia de infecciones intra-mama- 
Tabla 2. Análisis univariado entre nuevos casos de mastitis clínica y predictores agregados a nivel de vaca.

\begin{tabular}{|c|c|c|c|c|c|}
\hline FDR & CAT & $\mathrm{MCn}$ & $\mathrm{MC} \%$ & TOT & VAL \\
\hline \multirow{2}{*}{ NOP } & vaq & 13 & 4,3 & 300 & \multirow{2}{*}{0,0334} \\
\hline & $\mathrm{vac}$ & 47 & 8,2 & 576 & \\
\hline \multirow{3}{*}{ DPP } & arriba & 19 & 5,3 & 356 & \multirow{3}{*}{0,1226} \\
\hline & igual & 15 & 7,5 & 200 & \\
\hline & debajo & 16 & 10,3 & 155 & \\
\hline \multirow{4}{*}{$\mathrm{SDU}$} & 1 & 20 & 5,2 & 384 & \multirow{4}{*}{0,0164} \\
\hline & 2 & 14 & 7,1 & 198 & \\
\hline & 3 & 5 & 3,5 & 143 & \\
\hline & 4 & 23 & 11,1 & 207 & \\
\hline \multirow{2}{*}{ PEL } & no & 56 & 6,8 & 823 & \multirow{2}{*}{0,6088} \\
\hline & si & 6 & 5,5 & 109 & \\
\hline \multirow{3}{*}{ PDP } & normal & 53 & 7,2 & 736 & \multirow{3}{*}{0,8846} \\
\hline & seca & 8 & 6,9 & 116 & \\
\hline & grietas & 0 & 0 & 3 & \\
\hline \multirow{4}{*}{ APP } & $\mathrm{a}$ & 33 & 5,6 & 592 & \multirow{4}{*}{0,0001} \\
\hline & $b$ & 6 & 4,7 & 128 & \\
\hline & $\mathrm{c}$ & 8 & 6,2 & 129 & \\
\hline & $\mathrm{d}$ & 15 & 18,5 & 81 & \\
\hline \multirow{4}{*}{ HQT } & 1 & 19 & 4,4 & 436 & \multirow{4}{*}{0,0372} \\
\hline & 2 & 15 & 7,4 & 202 & \\
\hline & 3 & 11 & 8,4 & 131 & \\
\hline & 4 & 17 & 10,6 & 161 & \\
\hline \multirow{4}{*}{ CMT } & negat. & 15 & 4,0 & 378 & \multirow{4}{*}{0,0169} \\
\hline & traza & 18 & 7,3 & 248 & \\
\hline & uno & 12 & 7,6 & 157 & \\
\hline & dos & 17 & 11,4 & 149 & \\
\hline
\end{tabular}

Siglas horizontales: FDR: factor de riesgo, CAT: categoría, MCn: mastitis clínica (nuevos), MC\%: mastitis clínica (porcentajes), TOT: total, VAL: valor $\mathrm{px}$. Siglas verticales: NOP: número ordinal de partos, DPP: distancia de pezón a corvejón, SDU: suciedad de ubre, PEL: pérdida espontánea de leche, PDP: piel del pezón, APP: anillo punta pezón (a: ausencia, b: leve, c: moderado, d: extremo), HQT: hiperqueratosis (l: ausente, 2 : leve, 3 : mo-derado, 4 : extremo), CMT: california mastitis test (negativo traza, uno, dos).

rias ambientales y contagiosas ${ }^{22,23}$, como así también con el riesgo de mastitis clínica ${ }^{4}$.

En este estudio, vacas con ubres muy sucias mostraron mayor riesgo de mastitis clínica. En el mismo sentido, un estudio previo reportó que vacas con ubres clasificadas con grado 3 y 4 tenían un $50 \%$ más de chances de presentar MC ${ }^{4}$. En los sistemas lecheros con encierre a corral y en determinadas épocas del año, es común observar vacas que ingresan a la sala de ordeño con ubres sucias, lo cual representa un desafío para la glándula mamaria por el incremento en la exposición de la punta del pezón a patógenos ambientales.

El grado de suciedad de la ubre como un indicador del grado de limpieza del ambiente donde están las vacas ${ }^{23}$ podría ser utilizado como una herramienta de alerta en la toma de decisiones, considerando que las vacas deben permanecer en ambientes confortables, limpios y secos para asegurar una buena salud de ubre. Integrando el índice de suciedad en un enfoque pobla-
Tabla 3. Modelo de regresión logística para nuevos casos de mastitis.

\begin{tabular}{llccc}
\hline PRD & CAT & EE & valor $p$ & OR (IC 95\%) \\
\hline IC & \multicolumn{5}{c}{$-3,19(0,56)$} & $<0,0001$ \\
\hline DL & \multicolumn{5}{c}{$-0,0027(0,0013)$} & 0,0406 \\
\hline NP & vaq & referencia & \\
& vac & $-0,07(0,37)$ & 0,8389 & $0,93(0,45-1,93)$ \\
\hline SU & 1 & referencia & & \\
& 2 & $0,1(0,39)$ & 0,7977 & $1,11(0,51-2,37)$ \\
& 3 & $-0,63(0,54)$ & 0,2434 & $0,53(0,18-1,53)$ \\
& 4 & $0,94(0,39)$ & 0,0152 & $2,56(1,19-5,5)$ \\
\hline CMT & neg & referencia & & \\
& trz & $0,59(0,37)$ & 0,116 & $1,8(0,87-3,73)$ \\
& 1 & $0,45(0,44)$ & 0,306 & $1,57(0,66-3,72)$ \\
& 2 & $1,19(0,4)$ & 0,003 & $3,29(1,5-7,2)$ \\
\hline
\end{tabular}

PRD: predictor, IC: intercepto, DL: días de lactancia, NP: número de partos (ordinal), SU: suciedad de ubre, CMT: california mastitis test, CAT: categoría, vaq: vaquillona, vac: vaca, neg: negativo, trz: traza, EE: estimador

cional adquiere importancia la proporción de vacas sucias en el rodeo, respecto a este aspecto se sugiere que no debería ser superior el $15 \%{ }^{21}$, valor muy inferior al reportado en nuestro estudio.

El CMT es una herramienta diagnóstica para detectar infecciones subclínicas que se basa en la medición indirecta de células somáticas en la leche de cada cuarto, vacas con CMT negativo indican un nivel de células somáticas inferior a 200.000 células $/ \mathrm{mL}$. En este estudio, vacas con grado de CMT dos o más, presentaron un riesgo 3 veces mayor de presentar MC en los 15 días subsiguientes, con respecto a vacas negativas al CMT.

Si bien esta asociación no ha sido previamente reportada, en varias investigaciones se observó que vacas con historia de altos niveles de $\mathrm{MC}$ y recuento celular somático presentaban un mayor riesgo de experimentar otro evento de $\mathrm{MC}^{4,16,24}$, y este riesgo fue sustancialmente mayor si el caso de MC sucedía durante el mes previo. En este marco, disponer de la información de CMT nos permitió investigar su utilidad como predictor de casos nuevos de MC. Vacas con alto CMT previo a un caso clínico, podrían deberse a un estadio temprano de infección activa o bien a una infección subclínica que podría desarrollar a clínica, bajo circunstancias favorables.

La identificación de vacas con CMT 2 o más puede ser útil a los efectos de diseñar estrategias de segregación de vacas en ordeñe y a modo de facilitar un monitoreo más intensivo de las vacas que con más chances podrían adquirir nuevos episodios de MC. La fracción atribuible poblacional estimada del CMT y el índice de suciedad, $39 \%$ y $18 \%$ respectivamente, aportan evidencias cuantitativas de la importante fracción de los casos incidentes que podrían ser capturados a futuro con un monitoreo intensivo.

En nuestro estudio, a diferencia de lo reportado previamente ${ }^{4,17,20,24}$, el número de lactancias no fue una variable asociada al riesgo de MC. En otro estu- 
dio se analizaron separadamente vacas y vaquillonas, sin embargo, entre las vacas, aquellas de 3 o más partos tenían menor riesgo de $\mathrm{MC}$ que vacas más jóvenes. Asimismo, se observó un riesgo diferencial entre vacas y vaquillonas según el agente causal asociado a $\mathrm{MC}^{16}$.

En relación al estadio de lactancia, este factor ha sido previamente reportado con una tendencia similar a la observada en el presente estudio: a medida que avanza la lactancia la incidencia de MC es menor ${ }^{4,16,24}$. El estadio y el número de lactancias fueron incluidos en el modelo considerando que estos factores son fuentes potenciales de confusión al momento de ajustar el riesgo de $\mathrm{MC}$ por diferentes factores.

Las características del pezón, tal como fueron evaluadas en este estudio, no resultaron asociadas al riesgo de MC en contraposición a nuestra hipótesis. Sin embargo, otros estudios de similares características mostraron que grados severos de hiperqueratosis ${ }^{4} \mathrm{y}$ la pérdida espontánea de leche ${ }^{18}$ son factores que influyen aumentando el riesgo de $\mathrm{MC}$.

Si bien se encontró una asociación bivariada, al ajustar por el índice de suciedad y el grado de CMT, dichas variables resultaron no asociadas. Nuestros hallazgos de ninguna manera intentan minimizar el uso de estas mediciones como indicadores de salud mamaria, pero no deberían dejarse de lado factores potencialmente confundidores que pueden contribuir al riesgo de MC.

Los resultados de este estudio provienen de una muestra no aleatoria de tambos, por lo cual los datos reportados deben ser cuidadosamente extrapolados al resto de los tambos de la región. Además, los criterios de inclusión aplicados, tambos de manejo semi-pastoril y que aplicaban el plan de los 5 puntos de control de la mastitis, circunscribe las posibilidades de generalización, a cierto tipo de rodeos.

Respecto del sesgo de detección de MC entre tambos, dos de los investigadores que llevaron adelante el estudio, registraron todos los casos durante la primera visita y capacitaron al personal de ordeñe para la detección de casos y su registro durante los 15 días posteriores, razón por la cual se esperaría que la variación sistemática en la definición de caso, haya sido minimizada. Por otro lado, la variación en el error de medida del riesgo atribuida a la particularidad de cada rodeo, fue contemplada en el modelo, al incluir el tambo como efecto aleatorio.

El desafío de reducir los niveles de mastitis, entendiendo a ésta como una enfermedad multifactorial. debe ser enfocado de forma integral. A pesar de que no se dispone de estadísticas globales de la aplicación del plan del National Mastitis Council (NMC) en rodeos lecheros de Argentina, estos resultados sugieren el escenario de la mastitis clínica a partir del tipo de sistema y la aplicación del esquema de control convencional.

Los resultados de este estudio han revelado niveles de MC superiores respecto de reportes previos de Argentina. Los factores de riesgo identificados, CMT e índice de suciedad, serían responsables de una fracción significativa de los casos incidentes a corto plazo y por lo tanto, se constituyen en importantes indicadores de riesgo.

\section{REFERENCIAS}

1. Barkema HW et al. 1999. Management style and its association with bulk milk somatic cell count and incidence rate of clinical mastitis. J Dairy Sci 82: 1655-1663.

2. Barkema HW et al. 1998. Management practices associated with low, medium, and high somatic cell counts in bulk milk. J Dairy Sci 81: 1917-1927.

3. Bartlett PC, Miller GY, Lance SE, Heider LE. 1992. Environmental and managerial determinants of somatic cell counts and clinical mastitis incidence in Ohio dairy herds. Prev Vet Med 14: 195-207.

4. Breen JE, Green MJ, Bradley AJ. 2009. Quarter and cow risk factors associated with the occurrence of clinical mastitis in dairy cows in the United Kingdom. J Dairy Sci 92: 2551-2561.

5. DiRienzo JA et al. InfoStat versión 2018, Grupo InfoStat, FCA, Universidad Nacional de Córdoba, Argentina, http:// www.infostat.com.ar.

6. Gleeson DE, Meaney WJ, O'Callagham EJ, Rath MV. 2004. Effect of teat hyperkeratosis on somatic cell counts of dairy cows. Int J Appl Res Vet 2: 115-122.

7. Green MJ, Bradley AJ, Medley GF, Browne WJ. 2007. Cow, farm, and management factors during the dry period that determine the rate of clinical mastitis after calving. $J$ Dairy Sci 90: 3764-3776.

8. Halasa T, Nielen M, Huirne R, Hogeveen H. 2009. Stochastic bio-economic model of bovine intramammary infection. Livest Sci 124: 295-305.

9. Hogeveen H, Huijps K, Lam T. 2011. Economic aspects of mastitis: new developments. $N Z$ Vet J 59: 16-23.

10. Leelahapongsathon $\mathbf{K}$, Schukken $\mathbf{Y H}$, Suriyasathaporn W. 2014. Quarter, cow, and farm risk factors for intramammary infections with major pathogens relative to minor pathogens in Thai dairy cows. Trop Anim Health Prod 46: 1067-1078.

11. Llorca J, Fariñas C, Delgado RM. 2000. Fracción atribuible poblacional: cálculo e interpretación. Gac Sanit 15: 61-67.

12. Neave FK, Dodd FH, Kingwill RG, Westgarth DR. 1969. Control of mastitis in the dairy herd by hygiene and management. J Dairy Sci 52: 696-707.

13. Neijenhuis F, Barkema HW, Hogeveen H, Noordhuizen JP. 2000. Classification and longitudinal examination of callused teat ends in dairy cows. J Dairy Sci 83: 27952804.

14. National Mastitis Council (NMC). 2003. Current concepts of bovine mastitis (Bramley A.J.), 4th ed., Madison, USA.

15. Olde RG, Barkema HW, Kelton DF, Scholl DT. 2008. Incidence rate of clinical mastitis on Canadian dairy farms. J Dairy Sci 91: 1366-1377.

16. Oliveira CS. 2015. Cow-specific risk factors for clinical mastitis in Brazilian dairy cattle. Prev Vet Med 121: 297 305 
17. Pantoja JC, Almeida AP, Santos B, Rossi RS. 2016. An investigation of risk factors for two successive cases of clinical mastitis in the same lactation. Livest Sci 194: 1016.

18. Peeler EJ et al. 2000. Risk factors associated with clinical mastitis in low somatic cell count British dairy herds. $J$ Dairy Sci 83: 2464-2472.

19. Reneau JK, Seykora AJ, Heins BJ. 2003. Relationship of cow hygiene scores and SCC. Proc Natl Mast Coun (Madison, WI), pages 362-363.

20. Richardet M et al. 2016. Magnitud y variación de la mastitis clínica y sus costos asociados en rodeos lecheros de Argentina. Arch Med Vet 48: 153-158.
21. Ruegg PL. 2006. The role of hygiene in efficient milking. WCDS Advances in Dairy Technology 18: 285-293.

22. Santanna AC, Paranhos MJ. 2011. The relationship between dairy cow hygiene and somatic cell count in milk. $J$ Dairy Sci 94: 3835-3844.

23. Schreiner DA, Ruegg PL. 2003. Relationship between udder and leg hygiene scores and subclinical mastitis. $J$ Dairy Sci 86: 3460-3465.

24. Steeneveld $\mathbf{W}$ et al. 2008 . The influence of cow factors on the incidence of clinical mastitis in dairy cows. J Dairy Sci 91: 1391-1392.

25. Thrusfield M. 2007. Veterinary Epidemiology, 3rd ed., Blackwell Science, Oxford, UK, 593 p. 